\title{
MAGNETIC STUDIES OF POLYCRYSTALLINE MAGNETOSTRICTIVE CoPd FILMS WITH LARGE PLANAR STRAIN*
}

\author{
J. Dubowik and B. SzYmański \\ Institute of Molecular Physics, Polish Academy of Sciences \\ Smoluchowskiego 17, 60-179 Poznań, Poland
}

(Received Oclober 1, 1993)

\begin{abstract}
We report on ferromagnetic resonance and torque curves measurcments in electrodeposited $\mathrm{CoPd}$ alloy films with composition ranged from $\mathrm{Co}_{13} \mathrm{Pd}_{87}$ to $\mathrm{Co}_{45} \mathrm{Pd}_{55}$. We show that the origin of the multimode structure of ferromagnetic resonance spectra in these strongly magnetostrictive polycrystalline films can be satisfactorily explained on the basis of the independent-grain-approach for a textured microstructure. The fourfold periodicity of the torque curves for the compositional range of 30-35 at\% of Co seems to be originated by the microstructure features which lead to a distribution of the easy axis directions within the films.
\end{abstract}

PACS numbers: $75.50 . \mathrm{Kj}, 75.80 .+\mathrm{q}$

\section{Introduction}

The origin of the perpendicular anisotropy $\mathrm{K}_{\mathrm{U}}$ in $\mathrm{Co} / \mathrm{Pd}$ and $\mathrm{Co} / \mathrm{Pt}$ multilayers (MLs) has often been ascribed to magnetoelastic surface anisotropy [1]. In this aspect $\mathrm{Co} / \mathrm{Pd}$ MLs are particularly interesting, since the magnetostriction of CoPd is extremely large [2] and the films are subjected to a high tensile strain [3, 4]. In Co/Pd MLs, Co atoms in contact with Pd ones resemble CoPd in the sense of short range order and we may expect that they are similar to CoPd alloys in many aspects. For example, complicated ferromagnetic resonance (FMR) spectra of ultrathin Co films on Pd were observed [5] with large asymmetries and split peaks.

In this contribution, we report on the similar behaviour of both the FMR spectra and torque curves in CoPd electrodeposited alloy films. We show that the results of our studies can be explained on the basis of a model that takes into account the interplay between large magnetostriction, a planar tensile strain,

"This paper was presented at the European Conference "Physics of Magnetism 93", Poznan (Poland), 1993. 
and orientation of crystallites. In order to describe the line shapes of the FMR spectra we employ the independent-grain-approach proposed by Suran, Stankoff, and Hoffmann [6] who showed that, in polycrystalline films which are subjected to large strain, large anisotropic magnetostriction leads to a significant modification of FMR spectra. Here, we would like to outline some general ideas of this approach modified slightly by assuming a nonrandom distribution of the orientation of crystallites.

Generally, FMR absorption line shape can be described by

$$
P(H)=\frac{\Delta H}{8 \pi^{2}} \int_{0}^{\pi} \sin \alpha \mathrm{d} \alpha \int_{0}^{2 \pi} \frac{f(\alpha, \beta)}{\left(\frac{1}{2} \Delta H\right)^{2}+\left[H-H_{0}(\alpha, \beta)\right]^{2}} \mathrm{~d} \beta,
$$

where $H$ is the field applied normal to the film surface, $\Delta H$ is the line width and $H_{0}(\alpha, \beta)$ describes the resonance condition for a particular grain. $H_{0}(\alpha, \beta)$ includes the strain induced anisotropy fields $\lambda_{i} \sigma / M_{s}$, where $\sigma$ is the strain, $M_{\mathrm{s}}$ - the magnetization, and $\lambda_{i}$ stands for $\lambda_{100}$ or $\lambda_{111}$. The quantities $\alpha$ and $\beta$ are angles between the [001] and [100] cubic crystal axes and the sample normal, and $f(\alpha, \beta)$ is a function that describes the distribution of orientations of the crystallites. The resonance fields $H_{100}, H_{110}$, and $H_{111}$ present singularities for the stationary values where $\delta H_{0}(\alpha, \beta) / \delta \alpha=\delta H_{0}(\alpha, \beta) / \delta \beta=0$. The texture of the films described by the orientation distribution function $f(\alpha, \beta)$ influences intensity of FMR absorption at these singularities.

\section{Experimental details and results}

The films were prepared using electrodeposition technique onto glass substrates that had been coated first with $\mathrm{Cr}$ and $\mathrm{Cu}$ films by evaporation. Deposition was carried out from an unstirred electrolyte containing $\mathrm{Co}^{+2}$ and $\mathrm{Pd}^{+2}$ ions and ethylendiamine [4] at $55^{\circ} \mathrm{C}$ using the cathode current density $j$ ranged from $5 \mathrm{~mA} / \mathrm{cm}^{2}$ to $60 \mathrm{~mA} / \mathrm{cm}^{2}$. The composition of the films changed with $\mathrm{j}$ from $\mathrm{Co}_{13} \mathrm{Pd}_{87}$ to $\mathrm{Co}_{45} \mathrm{Pd}_{55}$ for $j=5$ and $60 \mathrm{~mA} / \mathrm{cm}^{2}$, respectively.

The structure of the films was examined by X-ray diffraction with Co $K_{\alpha}$ radiation at $36 \mathrm{kV}$ and $20 \mathrm{~mA}$. The diffractograms revealed a moderate [111]/[100] texture. Although no pole figures were measured, for future modelling of the FMR spectra we found it necessary to have at least an approximate picture of the grain orientations. The extent of the preferred orientation was evaluated from a comparison of diffractograms of the films with that of a powdered sample prepared by grinding several films which had previously been separated from the substrates. For the powdered sample that was regarded as a reference standard of randomly oriented CoPd crystallites, the intensity ratios of the three main reflections were $\left(I_{111} / I_{200} / I_{220}=100 / 15 / 14\right.$, for the $\mathrm{Co}_{22} \mathrm{Pd}_{78}$ film: $100 / 14 / 5$, and for the $\mathrm{Co}_{45} \mathrm{Pd}_{55}$ film: 100/8/4, respectively. The microstructure of the films changed gradually with decreasing the deposition current into a structure of more randomly oriented crystallites. According to our X-ray data, we propose to characterize the distribution of crystallites orientations by employing a two-dimensional function $f(\alpha, \beta)$ that is graphically depicted in Fig. 1 (inset) for description of a mixed $[111] /[100]$ texture in our $\mathrm{Co}_{45} \mathrm{Pd}_{55}$ film. The existence of the structure of this type seems to be the common situation in our electrodeposited CoPd films. 


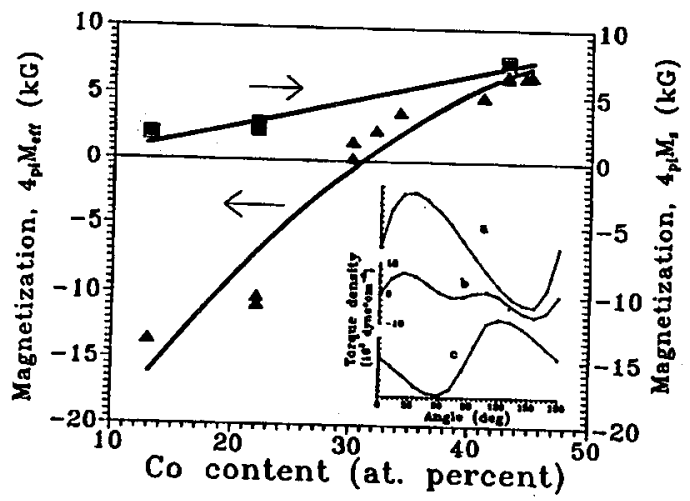

Fig. 1. FMR absorption of the $\mathrm{Co}_{45} \mathrm{Pd}_{55}$ (a) and $\mathrm{Co}_{30} \mathrm{Pd}_{70}$ (b) alloy films obtained by integration of the field derivative of FMR absorption. Dotted curves represent theoretical absorption spectra calculated according to (1) for two sets of parameters given in discussion. The inset shows the assumed orientation distribution function $f(\alpha, \beta)$ of crystallites in the $\mathrm{Co}_{45} \mathrm{Pd}_{55}$ film.

The FMR measurements were performed at $9 \mathrm{GHz}$ at room temperature in a microwave cavity which allowed us to vary the angle of the applied field with respect to the surface normal. The field derivative of FMR absorption was recorded. From the resonance positions taken for the static magnetic field applied parallel $\left(H_{\|}\right)$and perpendicular $\left(H_{\perp}\right)$ to the film plane, the $g$-factor and the effective magnetization $2 \pi M_{\mathrm{eff}}=4 \pi M_{\mathrm{s}}-H_{\mathrm{U}}\left(H_{\mathrm{U}}=2 K_{\mathrm{U}} / M_{\mathrm{s}}\right.$ is the perpendicular anisotropy field) were calculated using the standard resonance conditions for thin films [7].

The most striking feature observed in FMR spectra was their line shape. The FMR spectra were split into 2-3 branches (Fig. 1a) only over a restricted angular range of the field rotation angles (about $10^{\circ}$ ) from $H_{\perp}$. The splitting decreased with increasing the $\mathrm{Pd}$ content and, eventually, only a single mode was seen for the $\mathrm{Co}_{30} \mathrm{Pd}_{70}$ film (Fig. 1b). At $H_{\|}$only a single broad mode was observed for all the films. Since most of the spectra at $H_{\perp}$ were split the calculations of the $g$-factor and $4 \pi M_{\text {eff }}$ were carried in that way that we retained only those values which gave a $g$-factor value close to that of pure Co (2.18) due to lack of any data for CoPd alloys. Nevertheless, the values for the $g$-factor were scattered from 2.2 to 2.4. The similar significant distribution in $g$-values determined from FMR data were also observed in $\mathrm{Co} / \mathrm{Pt}$ [8] and Ni/Pd [9] MLs. No FMR absorption was observed for CoPd films with the Co content less than 30 at\%.

Torque measurements were performed in magnetic fields up to $15 \mathrm{kOe}$. The saturation magnetization $4 \pi M_{\mathrm{s}}$ was evaluated using the Chikazumi method [10]. From the compositional dependence of $4 \pi M_{\text {eff }}$ and $4 \pi M_{\mathrm{s}}$ (Fig. 2) it is seen that the absolute value of $H_{U}$ increases with decreasing Co content what agrees with the compositional dependence of $H_{U}$ in sputtered CoPd films [11].

Inset in Fig. 2 shows the torque curves taken at $13.5 \mathrm{kOe}$ rotating in a plane perpendicular to the film surface for $\mathrm{Co}_{22} \mathrm{Pd}_{78}$ (a), $\mathrm{Co}_{30} \mathrm{Pd}_{70}$ (b), and $\mathrm{Co}_{43} \mathrm{Pd}_{57}$ (c) films. For the films with Co content $>35$ at\% $H_{\mathrm{U}}<4 \pi M_{\mathrm{s}}$ and the normal to 


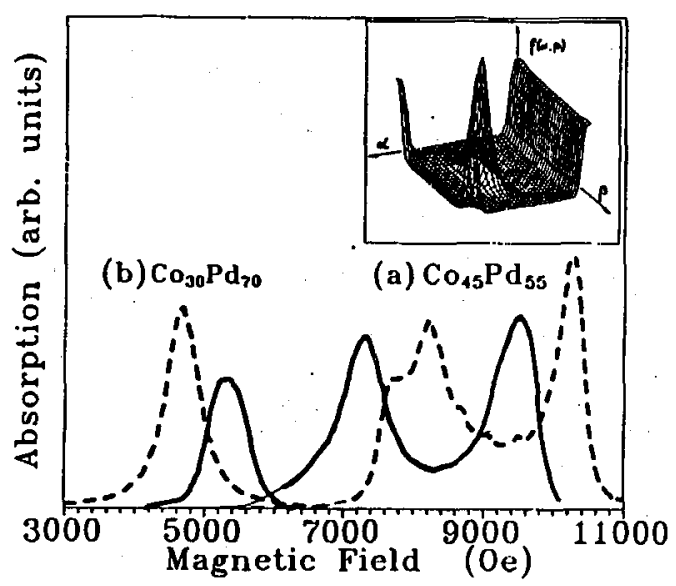

Fig. 2. The compositional dependence of the magnetization $4 \pi M_{\mathrm{s}}$ and the effective magnetization $4 \pi M_{\text {eff }}$ for $\mathrm{CoPd}$ films. The inset displays torque curves for the $\mathrm{Co}_{22} \mathrm{Pd}_{78}$ (a), $\mathrm{Co}_{30} \mathrm{Pd}_{70}$ (b), and $\mathrm{Co}_{43} \mathrm{Pd}_{57}$ (c) alloy films.

the film plane is the hard axis. For Co $<30$ at\%, $H_{\mathrm{U}}>4 \pi M_{\mathrm{s}}$ and the easy axis is perpendicular to the film surface. A characteristic behaviour of the torque curves for 30 at\% $<\mathrm{Co}<35$ at\% is seen in the inset (curve $-b$ ); the curve consists of a uniaxial term $(\propto \sin 2 \phi)$ and a biaxial one $(\propto \sin 4 \phi)$.

\section{Discussion}

In order to explain both the multimode nature of the FMR spectra and the two-term torque curves we have considered the interplay between the orientations of the crystallites in our films and the anisotropic magnetostriction $\left(\lambda_{100} \neq \lambda_{111}\right.$ for Co content > 20 at\% [2]) of CoPd alloy.

Our electrodeposited films are subjected to a high tensile strain [4] that results in large strain-induced anisotropy fields $H_{1}=3 \lambda_{100} \sigma / M_{\mathrm{s}} \neq H_{2}=3 \lambda_{111} \sigma / M_{\mathrm{s}}$. Since $H_{1}$ and $H_{2}$ differ substantially due to the anisotropic magnetostriction, the resonance fields corresponding to the singularities at $H_{100}, H_{110}$, and $H_{111}$ are distributed over a wide field range, according to (26), (27), and (28) in [6]. The FMR absorption calculated for the $\mathrm{Co}_{45} \mathrm{Pd}_{55}$ film, according to (1), is shown in Fig. 1a by a dotted curve. The following parameters were taken for calculations: the line width $\Delta H=200 \mathrm{Oe}, H_{1}=+900 \mathrm{Oe}, H_{2}=-2000 \mathrm{Oe}, M_{\mathrm{s}}=500 \mathrm{G}$, the frequency $f=9 \mathrm{GHz}$, and the distribution of grain-orientations depicted in the inset of Fig. 1. With decreasing the Co content in the CoPd films, the anisotropy of magnetostriction diminishes and, eventually, $\lambda_{100} \approx \lambda_{111}$ for $\mathrm{Co}_{22} \mathrm{Pd}_{78}$. The theoretical FMR absorption for the $\mathrm{Co}_{30} \mathrm{Pd}_{70}$ film (random distribution of the grain orientation is assumed) calculated with $\Delta H=500 \mathrm{Oe}, H_{1}=-1600 \mathrm{0e}$, $H_{2}=-2000 \mathrm{Oe}, M_{\mathrm{s}}=250 \mathrm{G}$, and $f=9 \mathrm{GHz}$, is shown in Fig. $1 \mathrm{~b}$ by a dashed curve. It seems that a fairly good qualitative agreement between the experimental absorption curves and the calculated ones was obtained despite a rather crude model of independent-grain-approach was considered. 
The peculiar behaviour of the torque curves may be tentatively explained taking into account a correlation between microstructure features and the strain induced anisotropy. Let us notice that when CoPd films have the composition with the Co content ranged from 25 to 35 at\%, where the effective magnetization $4 \pi M_{\mathrm{eff}} \approx 0\left(4 \pi M_{\mathrm{s}} \approx H_{\mathrm{U}}\right)$, then the torque exerted on the magnetization vector becomes very sensitive on the structural features related to the grain orientations. Because in this compositional range $\lambda_{100} \approx \lambda_{111}<0$ [2] and stress is tensile, both the (111) and (100) axes are easy axes of the strain-induced anisotropy for each grain. If we assume a mixed moderate $(111) /(100)$ texture or random distributions of the crystallites orientations, then we may regard the film as a system with a significant dispersion of easy axis. In effect, this may be the origin of the fourfold periodicity in the torque curves.

In conclusion, we have shown that magnetic properties of highly magnetostrictive CoPd alloy films may be satisfactorily explained by assuming the interplay between the microstructure and the high tensile strain.

Stimulating conversations with R. Gontarz are gratefully acknowledged. This work was supported by the grant No. 2291/2/91 of the Committee for Scientific Research.

\section{References}

[1] S. Tsunashima, K. Nakamura, II. Takahashi, S. Fukatsu, S. Uchiyama, Mater. Res. Soc. Symp. Proc. 231, 441 (1992).

[2] T. Tokunaga, M. Kohori, H. Kadomatsu, H. Fujiwara, J. Phys. Soc. Jpn. 50, 1411 (1981).

[3] B.N. Engel, C.D. England, R.A van Leeuwen, M.H. Wiermann, C.M. Falco, J. Appl. Phys. 70, 5873 (1991).

[4] R. Gontarz, L. Smardz, B. Szymański, P. Juzikis, J. Magn. Magn. Maler. 120, 278 (1993).

[5] S.T. Purcell, II.W. van Kesteren, E.C. Cosman, W.B. Zeper, W. Hoving, J. Appl. Phys. 69, 5640 (1991).

[6] G. Suran, A. Stankoff, F. Hoffmann, Phys. Rev. B 8, 1109 (1972).

[7] A.Z. Maksymowicz, L.J. Maksymowicz, J.S.S. Whiting, Acta Phys. Pol. 80, 665 (1991).

[8] Z. Zahng, P.E. Wigen, S.S.P. Parkin, J. Appl. Phys. 69, 5649 (1991).

[9] N.K. Flevaris, R. Krishnan, J. Magn. Magn. Mater. 93, 439 (1991).

[10] S. Chikazumi, J. Appl. Phys. 32, 81S (1961).

[11] S. Hashinoto, Y. Ochiai, K. Aso, Jpn. J. Appl. Phys. 28, 1596 (1989). 